\title{
Síndrome de Plummer-Vinson: uma rara associação na talassemia
}

\section{Plumer-Vinson syndrome: A rare association with thalassemia}

Eduardo Crema ${ }^{1}$

Ana Marcela R. Fonseca ${ }^{2}$

Lara Beatriz P. Ribeiro ${ }^{3}$

Cecília Aparecida D. Bello

Paulo Roberto J. Martins ${ }^{4}$

Alex Augusto Silva ${ }^{5}$

\begin{abstract}
A síndrome de Plummer-Vinson é caracterizada por disfagia cervical, deficiência de ferro e presença de membrana esofágica. Neste estudo, relatam-se dois casos dessa síndrome em irmãos adolescentes. Eles não obtiveram aumento dos níveis hematimétricos após reposição com ferro oral, o que, associado à eletroforese de hemoglobinas, sustentou o diagnóstico de talassemia concomitante. Devido ao quadro dos filhos, os pais foram também submetidos à eletroforese de hemoglobinas cujo diagnóstico do pai foi talassemia alfa/beta menor e da mãe, talassemia alfa menor. Os irmãos tiveram disfagia refratária e necessitaram de dilatação endoscópica. Ambos necessitaram de terapia com ferro venoso com melhora dos níveis hematimétricos. Rev. bras. hematol. hemoter. 2007;29(4):412-415.
\end{abstract}

Palavras-chave: Síndrome de Plummer-Vinson; disfagia; talassemia; anemia ferropriva.

\section{Introdução}

A síndrome de Plummer-Vinson, também conhecida como síndrome de Patterson-Kelly, caracteriza-se por disfagia cervical, deficiência de ferro e presença de membrana esofágica. É uma síndrome rara, incomum antes de 30 anos $^{1} \mathrm{e}$ afeta, principalmente, mulheres brancas, entre 40 e 70 anos. Na literatura, verificam-se poucos relatos sobre sua ocorrência em crianças ou adolescentes. ${ }^{2,3}$

Alguns autores apresentam essa síndrome associada a processos auto-imunes, como artrite reumatóide, anemia perniciosa, tireoidite, e também à predisposição genética, ${ }^{4,5,6}$ doença celíaca, ${ }^{7}$ mucosa gástrica ectópica ${ }^{8}$ e a fatores nutricionais e infecciosos. ${ }^{9}$ São raras as citações sobre PlummerVinson associada à talassemia.

Neste estudo são relatados dois casos de adolescentes irmãos com síndrome de Plummer-Vinson associada à talassemia alfa.

A primeira adolescente, desde 1 ano de idade, portava anemia, tratada com ferro oral e ácido fólico sem sucesso. Nessa época, foi feita eletroforese de hemoglobina, que diagnosticou talassemia alfa. Aos 3 anos iniciou disfagia intermitente a alimentos sólidos e, por uma epigastralgia aos 11 anos, submeteu-se à endoscopia digestiva alta, quando foi descoberta membrana pré-cricóide e estabelecido diagnóstico de Plummer-Vinson.

O segundo adolescente também iniciou anemia com um ano de idade, sem resolução com o uso de ferro oral e ácido fólico. Foi submetido à eletroforese de hemoglobina que, tal como sua irmã, apresentou talassemia alfa. Com a mesma idade, 3 anos, surgiu disfagia intermitente a alimentos sólidos e, por ter sintomas semelhantes, suspeitou-se de Plummer-Vinson, o qual foi confirmado por esofagograma aos 6 anos de idade.

Em virtude do quadro dos filhos, os pais também foram submetidos à eletroforese de hemoglobina, a qual apresentou talassemia alfa/beta minor para o pai e talassemia alfa minor para a mãe.

\footnotetext{
${ }^{1}$ Professor titular do Serviço de Cirurgia do Aparelho Digestivo - UFTM.

${ }^{2}$ Médica hematologista e do Hemocentro Regional de Uberaba - UFTM.

${ }^{3}$ Aluna de Graduação da Medicina - UFTM.

${ }^{4}$ Professor adjunto da Disciplina de Hematologia e coordenador do Hemocentro Regional de Uberaba - UFTM.

${ }^{5}$ Professor adjunto e chefe do Serviço de Cirurgia do Aparelho Digestivo - UFTM.
}

Correspondência: Eduardo Crema

Rua Marcos Lombardi, 305

38050-170 - Uberaba-MG - Brasil

Tel.: (34) 3318-5228; Fax: 3318-5288

E-mail:cremauftm@mednet.com.br 


\section{Caso 1}

Paciente de 17 anos, sexo feminino, procurou o departamento de cirurgia do aparelho digestivo, em dezembro de 2004. Queixava-se de disfagia cervical para alimentos sólidos, intermitente, desde os 3 anos de idade; cansaço aos grandes esforços e hipermenorréia há três anos, controlada há um ano com o uso de contraceptivo oral. Referia ser portadora de talassemia, diagnosticada no seu primeiro ano de vida, pela eletroforese das hemoglobinas. Ao exame físico apresentava mucosas descoradas $(2+/ 4+)$, anictéricas, sem visceromegalias.

Os exames laboratoriais mais recentes revelaram: níveis de hemoglobina de $7,2 \mathrm{~g} / \mathrm{dL}$, hematócrito $28 \%$, volume corpuscular médio (VCM) 61fL, HCM 16pg, leucócitos $6.200 / \mathrm{mm}^{3}$, plaquetas $500.000 / \mathrm{mm}^{3}$, vitamina B12 388pg/ml, ácido fólico $21 \mathrm{ng} / \mathrm{ml}$, ferritina $7 \mathrm{ng} / \mathrm{ml}$, ferro $12 \mathrm{mcg} / \mathrm{dL}$, TIBC 632mg/dl, índice de saturação 2\%. Eletroforese de hemoglobina: AH, hemoglobina $A_{2} 2,29 \%$, hemoglobina fetal 0,5\%, hemoglobina $\mathrm{H} 0,6 \%$.

Desde cerca de 1 ano de idade, essa paciente recebia terapia oral com ferro e ácido fólico, sem melhora dos níveis séricos de hemoglobina, ferro e ferritina. Aos 11 anos foi submetida à endoscopia digestiva alta (EDA) para avaliação de epigastralgia, quando se verificou ausência de progressão do endoscópio. Foi realizado um esofagograma que mostrou membrana esofágica na altura do músculo cricofaríngeo e estabeleceu-se o diagnóstico de síndrome de Plummer Vinson.

Por conseguinte, a paciente foi submetida novamente à EDA e confirmou-se a presença da membrana em região pré-cricóide, sem outros achados patológicos do esôfago ou estômago. Realizou-se a ruptura endoscópica da membrana e a paciente permaneceu assintomática até os 16 anos. Em virtude do retorno progressivo do quadro disfágico, uma nova ruptura foi necessária. Não houve progressão do endoscópio e o esofagograma confirmou estenose em esôfago cervical (Figura 1).

Aos 17 anos, a paciente apresentou-se novamente no serviço de cirurgia do aparelho digestivo, onde se realizou uma EDA. Esse exame revelou subestenose de esôfago proximal associada à esofagite distal, erosiva, grau 1. Então, a paciente foi submetida à dilatação com sonda de Savary e, para excluir doença celíaca, solicitou-se dosagem dos anticorpos antiendomiso e antigliadina: ambos negativos.

Depois de quatro anos sem medicação, a terapia oral com ferro e ácido fólico foi reintroduzida. Após dois meses de reposição, os exames laboratoriais não revelaram melhora do metabolismo do ferro. Desse modo, optou-se pela reposição endovenosa, cujos resultados apresentam-se na tabela 1. Clinicamente, a paciente permaneceu sem disfagia. $O$ esofagograma pós-dilatação revelou melhora do calibre do esôfago proximal e boa passagem do contraste (Figura 2). Esse aspecto radiológico assemelha-se ao do seu irmão, conforme se relata a seguir.

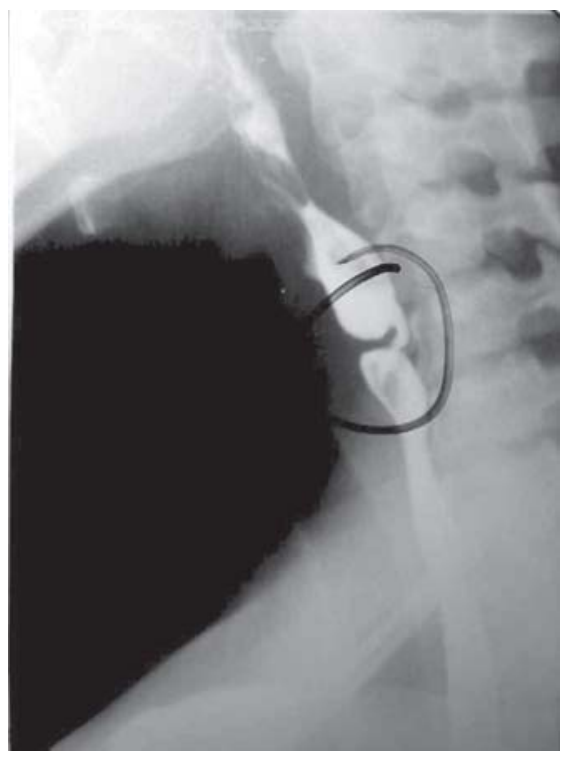

Figura 1.

Esofagograma, na pré-dilatação, que evidencia estenose em esôfago cervical

Tabela 1. Caso 1 - Exames laboratoriais durante a reposição do ferro

\begin{tabular}{|c|c|c|c|c|}
\hline & & $\begin{array}{l}\text { Antes do } \\
\text { ferro } \\
\text { via oral }\end{array}$ & $\begin{array}{c}\text { Após o } \\
\text { ferro } \\
\text { via oral }\end{array}$ & $\begin{array}{l}\text { Após o ferro } \\
\text { endovenoso }\end{array}$ \\
\hline \multirow{8}{*}{ Caso 1} & $\begin{array}{l}\text { Hemácias } \\
\left(\times 10^{12} / I\right)\end{array}$ & 4,56 & 4,15 & 4,65 \\
\hline & Hemoglobina (g/dl) & 7,2 & 7,2 & 15 \\
\hline & Hematócrito (\%) & 28,0 & 25,1 & 43,3 \\
\hline & VCM (fl) & 61 & 60 & 93 \\
\hline & Ferritina (ng/ml) & 7,0 & 4,0 & 213,3 \\
\hline & $\begin{array}{l}\text { Plaquetas } \\
\text { (x 109\%) }\end{array}$ & 5,00 & 4,41 & 2,18 \\
\hline & $\begin{array}{l}\text { Leucócitos } \\
\left(\times 10^{3} / \mathrm{mm}^{3}\right)\end{array}$ & 6,20 & 8,60 & 7,05 \\
\hline & $\begin{array}{c}\text { Hemoglobina A2 } \\
(\%)\end{array}$ & 2,29 & 1,70 & 2,70 \\
\hline
\end{tabular}

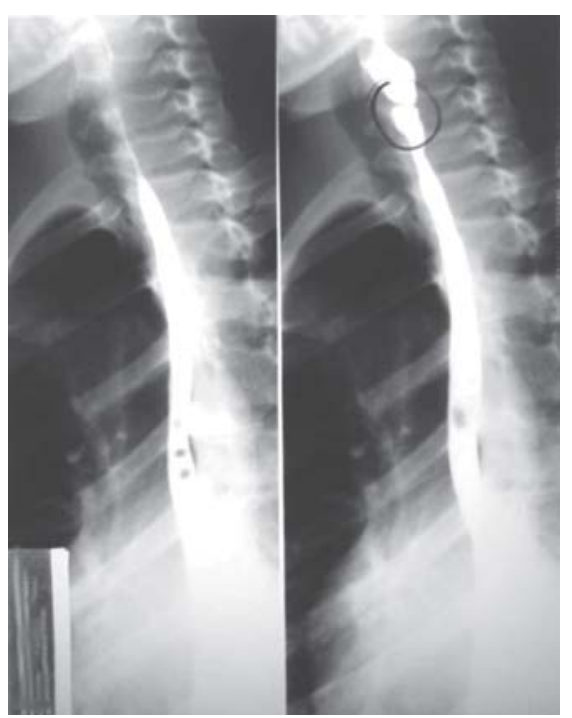

Figura 2.

Esofagograma, na pós-dilatação, que evidencia calibre normal do esôfago cervical 


\section{Caso 2}

Paciente de 12 anos, sexo masculino, procurou o departamento de cirurgia do aparelho digestivo em dezembro de 2004, juntamente com a irmã, caso 1. Queixava-se de disfagia cervical para alimentos sólidos, intermitente, desde os 3 anos e cansaço aos grandes esforços. Referia ser portador de talassemia, também diagnosticada com um ano de idade pela eletroforese das hemoglobinas. Ao exame físico, apresentava mucosas descoradas $(2+/ 4+)$, anictéricas, queilite angular, língua geográfica e baço palpável a quatro $\mathrm{cm}$ do rebordo costal esquerdo.

Os exames laboratoriais revelaram: níveis de hemoglobina de 6,9g/dL, hematócrito 24,7\%, volume corpuscular médio (VCM) 48,5fL, HCM 13,5pg, leucócitos 5.800/.mm33, plaquetas $550.000 / \mathrm{mm}^{3}$, vitamina B12 413pg/ml, ácido fólico 21ng/ml, ferritina 19ng/ml TIBC 509mg/dl, índice de saturação 2\%. Eletroforese de hemogobina: AH, hemoglobina $\mathrm{A}_{2}$ $3,46 \%$, hemoglobina fetal 0,3\%, hemoglobina $\mathrm{H} 0,5 \%$.

O paciente apresentava níveis séricos de hemoglobina, ferro e ferritina abaixo do normal, desde lactente, sem melhoras com reposição oral de ferro e ácido fólico. O diagnóstico de Plummer Vinson, suspeitado em virtude de quadro semelhante ao da irmã, foi estabelecido aos 6 anos de idade pelo esofagograma. Nesta época, o paciente foi submetido à ruptura endoscópica da membrana e, aos 8 anos, à segunda ruptura.

Em virtude do retorno progressivo do quadro disfágico aos 12 anos, o paciente apresentou-se no departamento de cirurgia do aparelho digestivo. A endoscopia digestiva alta mostrou estenose de esôfago proximal. Por conseguinte, foi submetido à dilatação endoscópica com sonda de Savary e, como no caso 1, solicitou-se dosagem dos anticorpos antiendomiso e antigliadina, ambos com resultado negativo.

Depois de quatro anos sem medicação, foi reintroduzida a terapia oral com ferro e ácido fólico. Após dois meses de reposição, não houve resposta à medicação. Também foi feita a ferroterapia endovenosa, cujos resultados apresentamse na tabela 2. Clinicamente, o paciente permanece sem disfagia. Os aspectos radiológicos pré e pós-dilatação foram semelhantes aos de sua irmã.

\section{Antecedentes}

Devido ao quadro dos filhos, os pais foram submetidos ao exame de eletroforese de hemoglobina. O pai sempre foi assintomático. Seu diagnóstico foi descoberto ao se constatarem os seguintes resultados desse exame: $\mathrm{AH}$, hemoglobina $A_{2} 5,27 \%$, hemoglobina fetal 0,2\%, hemoglobina $H$ $0,4 \%$, resistência osmótica 0,35 de $87 \%$. Diante disso, diagnosticou-se talassemia alfa/beta menor.

A mãe refere ter apresentado anemia quando esteve grávida, porém, atualmente, mantém níveis de hemoglobina normais. O resultado de sua eletroforese foi: $\mathrm{AH}$, hemoglobina
Tabela 2. Caso 2 Exames laboratoriais durante a reposição do ferro

\begin{tabular}{|c|c|c|c|c|}
\hline & & $\begin{array}{l}\text { Antes do } \\
\text { ferro } \\
\text { via oral }\end{array}$ & $\begin{array}{c}\text { Após o } \\
\text { ferro } \\
\text { via oral }\end{array}$ & $\begin{array}{l}\text { Após o ferro } \\
\text { endovenoso }\end{array}$ \\
\hline \multirow{8}{*}{ Caso 2} & $\begin{array}{l}\text { Hemácias } \\
\left(\times 10^{12} / I\right)\end{array}$ & 5,10 & 5,75 & 6,26 \\
\hline & Hemoglobina (g/dl) & 6,9 & 7,7 & 10,3 \\
\hline & Hematócrito (\%) & 24,7 & 27,8 & 33,6 \\
\hline & VCM (fl) & 48,5 & 48,3 & 53,7 \\
\hline & Ferritina (ng/ml) & 19,0 & 4,3 & 122,3 \\
\hline & $\begin{array}{l}\text { Plaquetas } \\
\text { (x 10\%/l) }\end{array}$ & 5,50 & 6,57 & 4,85 \\
\hline & $\begin{array}{l}\text { Leucócitos } \\
\left(\times 10^{3} / \mathrm{mm}^{3}\right)\end{array}$ & 5,80 & 6,89 & 5,68 \\
\hline & $\begin{array}{c}\text { Hemoglobina A2 } \\
(\%)\end{array}$ & 3,46 & 1,6 & 2,1 \\
\hline
\end{tabular}

$\mathrm{A}_{2} 2,8 \%$, hemoglobina fetal $0,1 \%$, hemoglobina $\mathrm{H} 1,0 \%$. Concluiu-se: talassemia alfa menor.

\section{Discussão}

A etiopatogenia da Síndrome de Plummer-Vinson é desconhecida. Neste estudo, a presença da mesma síndrome em irmãos sustenta a hipótese da predisposição familiar relatada por Atmatzidis et al. ${ }^{6} \mathrm{O}$ papel preciso da deficiência de ferro, no desenvolvimento da síndrome de Plummer Vinson, é controverso.

Alguns autores acreditam que a redução das enzimas oxidativas dependentes de ferro resulta em degradação dos músculos da faringe e atrofia da mucosa e, consequëntemente, desenvolvimento de membranas. ${ }^{6}$ Entretanto, a ferropenia, além da disfagia cervical e da membrana esofágica superior, faz parte da tríade clássica do Plummer Vinson.

Os exames laboratoriais dos pacientes foram compatíveis com ferropenia e, juntamente com o esofagograma e a endoscopia, propiciaram o diagnóstico da síndrome. O esofagograma, principalmente em incidência lateral, associado ao cinedeglutograma, constituiu um método bastante sensível para mostrar a membrana no esôfago cervical. ${ }^{6,10}$

As membranas são mucosas finas (1mm a $2 \mathrm{~mm}$ ), raramente circunferenciais ou múltiplas, ${ }^{1}$ que se localizam abaixo dos músculos cricofaríngeos, na parede anterior do esôfago, e estendem-se lateralmente. Na totalidade dos casos, o diagnóstico é feito pela endoscopia digestiva alta. ${ }^{10}$

Classicamente, a talassemia cursa com níveis de ferro normal ou elevado, logo, não justificaria o desenvolvimento da síndrome de Plummer-Vinson, nos casos apresentados. No entanto, a associação de talassemia com anemia ferropriva, apesar de rara, é relatada. Steinberg et al. ${ }^{11}$ verificaram que, em 47 indivíduos portadores de talassemia alfa, os parâmetros microcíticos hipocrômicos deviam-se à combinação entre deficiência de ferro e talassemia alfa. Em outros casos anali- 
sados, preocupou-se em excluir a associação com doença celíaca, conforme se verifica em outro estudo. ${ }^{7}$ Observa-se a importância de se investigarem outras causas de anemia e, particularmente, frente a uma anemia microcítica, excluir talassemia, uma vez que esta e a ferropenia são os principais exemplos desse tipo de microcitose.

Neste estudo, o não-aumento dos níveis hematimétricos, após reposição com ferro oral, associado à eletroforese de hemoglobinas, sustenta o diagnóstico de talassemia concomitante. No entanto, quando se realizou a reposição endovenosa do ferro, houve melhora da resposta, o que evidenciou uma incapacidade parcial de absorção intestinal do ferro, em ambos os casos.

A nova dosagem de hemoglobina $\mathrm{A}_{2}$ e os níveis hematimétricos, após a reposição endovenosa de ferro, confirmaram o diagnóstico de talassemia alfa menor, no caso 1, e talassemia alfa / beta menor, no caso 2 .

Quanto à terapia da síndrome de Plummer-Vinson, em alguns pacientes, a melhora da disfagia ocorre com a reposição via oral de ferro. ${ }^{6}$ Quando essa terapia não é eficiente, há necessidade de ruptura da membrana endoscopicamente, ou, inclusive, de dilatações para melhora do quadro. ${ }^{1}$

Há um estudo que preconiza as dilatações esofágicas como tratamento de escolha. ${ }^{10} \mathrm{Em}$ alguns casos, é necessário repetir essas dilatações. ${ }^{1,3}$ Na casuística de Cecconello et al., ${ }^{10} 50 \%$ dos pacientes necessitaram de mais de uma dilatação endoscópica para resolução do quadro de disfagia.

Neste relato, a luz esofágica dos pacientes - casos 1 e 2 - estava bastante obstruída pela membrana e foi necessária a ruptura endoscópica e, posteriormente, a dilatação, em virtude do retorno do quadro de disfagia.

Em relação à terapia para a anemia ferropriva, foi proposta, inicialmente, a reposição de ferro via oral. A ausência de resposta conduziu à terapia endovenosa. Não há dúvidas de que esses pacientes deverão ser acompanhados regularmente, com infusões endovenosas periódicas de ferro, com vista a, inclusive, evitar recidivas das membranas cricofaríngeas.

Além disso, é importante o seguimento prolongado dos portadores da Síndrome de Plummer-Vinson mediante realização de endoscopias periódicas, pois essa síndrome é considerada uma condição pré-cancerosa. ${ }^{3,12}$ Cerca de $10 \%$ dos pacientes desenvolvem câncer de esôfago ou de faringe. ${ }^{12}$ Com base neste relato, ressalta-se a importância de se investigarem doenças associadas nos portadores de PlummerVinson.

\section{Abstract}

Plummer-Vinson syndrome is characterized by cervical dysphagia, iron deficiency and the presence of esophageal membranes. We report two cases of this syndrome present in adolescent brothers with associated thalassemia. After oral iron therapy, their hematimetric levels showed no increase, which associated with the results of hemoglobin electrophoresis, sustained the diagnosis of thalassemia. Due to the condition of the children, the parents were submitted to hemoglobin electrophoresis examinations; the father was diagnosed as having minor alpha/beta thalassemia and the mother as minor beta thalassemia. Both patients suffered from refractory dysphagia and required endoscopic dilatation. They both underwent venous iron therapy, which improved the hematimetric levels. Rev. bras. hematol. hemoter. 2007;29(4):412-415.

Key words: Plummer-Vinson syndrome; dysphagia; thalassemia; iron deficiency anemia.

\section{Referências Bibliográficas}

1. Haygert CJP, Marconcini JF, Marconato MC, Melo LO, Antunes PSP, Baladão JCS. Síndrome de Plummer-Vinson. JBM. 2000; 79(3):46-51.

2. Ninomiya DI, Cichowolski V, Castagnino N, Sica G, Dillon MH. Síndrome de Plummer-Vinson en Pediatría. Arch. Argent. Pediatr. 2001;99(6):534-7.

3. Anthony R, Sood S, Strachan DR, Fenwick JD. A case of PlummerVinson syndrome in childhood. Journal of Pediatric Surgery. 1999; 34(10):1570-2.

4. Makharia GK, Nandi B, Garg PK, Tandon RK. Plummer-Vinson syndrome: unusual features. Indian J Gastroenterol.2002;21:74-5.

5. Malhotra P, Kochhar R, Varma N, Kumari S, Jain S, Varma S. Paterson-Kelly syndrome and celiac disease - a rare combination. Indian J Gastroenterol. 2000;19:191-2.

6. Atmatzidis K, Papaziogas B, Pavlidis T, Mirelis Ch, Papaziogas T. Plummer-Vinson syndrome. Diseases of the Esophagus. 2003; 16:154-7.

7. Dickey W, McConnell B. Celiac disease presenting as the PatersonBrown Kelly (Plummer-Vinson) syndrome. AJG. 1999;94(2):527-9.

8. Weaver G. Upper esophageal web due to a ring formed by squamocolumnar junction with ectopic gastric mucosa. Dig Dis Sci. 1979;21:959-63.

9. Wynder E, Fryer J. Etiologic considerations of Plummer-Vinson (Patterson-Kelly) syndrome. Ann Intern Med. 1958;49:1106-28.

10. Cecconello I, Felix VN, Zilberstein B, Machado MAC, Volpe P, Pinotti HW. Membrana esofágica cervical e síndrome de PlummerVinson: apresentação de casuística e revisão da literatura. Rev. Hosp. Clín. Fac. Med. S. Paulo. 1994;49(4):148-51.

11. Steinberg MH, Forget BG, Higgs DR, Nagel RL. Disorders of hemoglobin. Cambridge University Press, Cambridge, UK, 2001.

12. Messmann H. Squamous cell cancer of the oesophagus. Best Practice \& Research Clinical Gastroenterology. 2001;15(2):249-65.

Avaliação: Editor e dois revisores externos

Conflito de interesse: não declarado

Recebido: 29/06/2006

Aceito após modificações: 12/04/2007 